ORIGINAL PROF-2265

\title{
PULMONARY TUBERCULOSIS;
}

\section{Frequency of smear positive in private laboratories of Sargodha outside the TB-DOTS programme}

Dr. Saeed Akram Bhatti, Dr. Abdul Haseeb Khan, Dr. Maqbool Alam

ABSTRACT... Objective: To assess the frequency of smear positive pulmonary tuberculosis patients diagnosed at private laboratories of Sargodha not included in the national DOTS (Direct Observational Therapy Short Course) programme. Study Design: A retrospective study. Place and Duration of Study: Private laboratories of Sargodha from January 2007 to December 2012. Methodology: Data analysis of 3952 patients who reported to private laboratories of Sargodha for staining of sputum for AFB was carried out. They were all self reporting and were either suspects of pulmonary tuberculosis or had a history of contact with a smear positive case of TB. Staining was done by Ziehl-Neelson method. The smear results were reported as positive or negative. Results: Out of total 3952 specimens 373 $(9.21 \%)$ were found to be positive for the presence of acid fast bacilli. From these 373 positive cases 164 (44\%) were female and 209 (56\%) were males. The age group most frequently associated with the smear positive result was aged $>55$ years $(13.89 \%)$ followed by age $<15$ years $(10 \%)$. Conclusions: A large number of smear positive patients are diagnosed in private sector. These patients are not registered in DOTS programme of World Health Organization. Their number is not included in the calculating the prevalence TB in our country. It is recommended that TB case notification should be made mandatory by law by private sector health care providers.

Key words: Pulmonary tuberculosis, Frequency, Mycobacterium tuberculosis, Sargodha

Article Citation

Bhatti SA, Khan AH, Alam M. Pulmonary tuberculosis; frequency of smear positive in private laboratories of Sargodha outside the TBDOTS programme. Professional Med J 2013;20(6): 887-890.

\section{INTRODUCTION}

In Pakistan the first TB survey was carried out in 1962. A twenty years TB control programme was then initiated that focused on establishing specialized TB centers and special TB wards at the DHQ hospitals ${ }^{1}$. Because of the serious public health threat WHO declared tuberculosis as a global emergency in 1993. Pakistan was one of the countries that showed a sluggish and inadequate response to control tuberculosis. In 1998 Pakistan was declared as one of the 16 countries struggling to control TB ${ }^{2,3}$. DOTS was started in Pakistan from 1995. A real progress was made in 2001 when TB was declared as national emergency through Islamabad declaration. To achieve its objective and targets National TB control programme ${ }^{4}$ has introduced a standard system of registration and reporting. In accordance with the WHO recommendations a series of standardized records are created including TB treatment card, TB appointment card, District TB register, TB laboratory register, TB smear examination request report form, TB culture/sensitivity test. The case finding, smear conversions at follow up, and final results of treatment are collected at all levels of the health system on quarterly basis and a cohort analysis is carried out. WHO estimates of the TB burden of the country is based on these reports. Pakistan is still in top 22 countries with high TB burden, ${ }^{5,13}$. In its report of 2012 WHO has calculated prevalence rate of 350/100,000. The smear positive cased recorded were 105733 $(41 \%)^{5}$. The epidemiological data collected through the national TB control programme from all levels of public health system is reliable and suitable for programme management. We have carried out a survey to assess the involvement of private health care providers in national TB control programme. This study has revealed that substantial numbers of cases of sputum positive cases are not recorded in the national TB control programme.

\section{METHODS AND MATERIAL}

A data was collected from the local laboratories in the urban area of Sargodha. Majority of laboratories had very poor record keeping and they were excluded from the study. The study period extended from January 2007 to December 2012. They did not represent any 


\begin{tabular}{|l|c|c|c|c|}
\hline \multicolumn{1}{|c|}{ Characteristics } & Total examined & Smear positive & \%age & p-value \\
\hline Gender & & & & \multirow{2}{*}{$\mathrm{p}=0.24$} \\
\hline Male & 2327 & 209 & 8.98 & 10.09 \\
\hline Female & 1625 & 164 & 9.44 & \\
\hline Total & 3952 & 373 &
\end{tabular}

Table-I. Distribution of smear positive pulmonary TB prevalence rate by gender

specific socioeconomic group or specific area of population. Empty containers (labeled) were given to the patients. The proper procedure of sputum collection was explained. They were instructed and demonstrated how to breathe deeply and cough to bring up the sputum and not the saliva in the container. In majority of the patients an early morning specimen was preferred. However sputum was also collected at time of presentation in the laboratory. Sputum staining was done by laboratory technologists who have been given training on how to stain and detect the acid fast bacilli. Staining was done by Ziehl-Neelson method. The smear results were reported as positive or negative.

The data collected was entered in a predesigned proforma indicating age and sex of the patient, total number of case and smear positive case. The proportions of smear positive cases for the overall AFB screened were determined for each year. The distributions of smear positive cases between gender and among different age groups were tabulated. Statistical analysis was then carried out for $p$-value

\section{RESULTS}

A total number of 3952 sputum smear were stained by Ziehl-Neelson method for the detection of Acid Fast bacilli from January 2007 to December 2012 (6 years). Out of these 1625 (41.1\%) were females and 2327 (58.9) were males. Although more number of male patients reported for sputum examination, 2327 as compared to females1625, however the percentage of smear positive cases were more in females 164 (10.09\%) as compared to 209 (8.89\%) males. However, statistically significant difference was not observed between gender and sputum positive pulmonary tuberculosis $(P=0.24)$ (Table-I)

Those showing the positive results were divided into four age groups. The age group most frequently associated with the smear positive result was aged $>$ 55 years, followed by age group of $<15 y e a r s$. Smear positive pulmonary tuberculosis prevalence distribution is significantly different among classes of age groups $(P<0.001)$ (Table-II).

The maximum number of patients examined for AFB was in the year 2009 (810), however the number of positive smear was the minimum 73 (9.01\%). The number of patients examined in the years 2007 and 2012 was 550 and the positive cases were 52 (9.45\%) and $9.21 \%$ respectively. The number of positive pulmonary tuberculosis has been persistently above 9 $\%$ (range 550-810) (52-73) during the period from January 2007 to December 2012 (Table-III).

\section{DISCUSSION}

Under the national TB control programme ${ }^{3}$, a well organized system with a network of diagnostic centers and treatment centers has been established at different levels including BHUs (basic health units), RHCs (rural health centers) and TB hospitals where specialist advice is available. Periodic workshops are held for training of the doctors and paramedical staff. The TB cases are detected, registered and reported to $\mathrm{WHO}$ in 


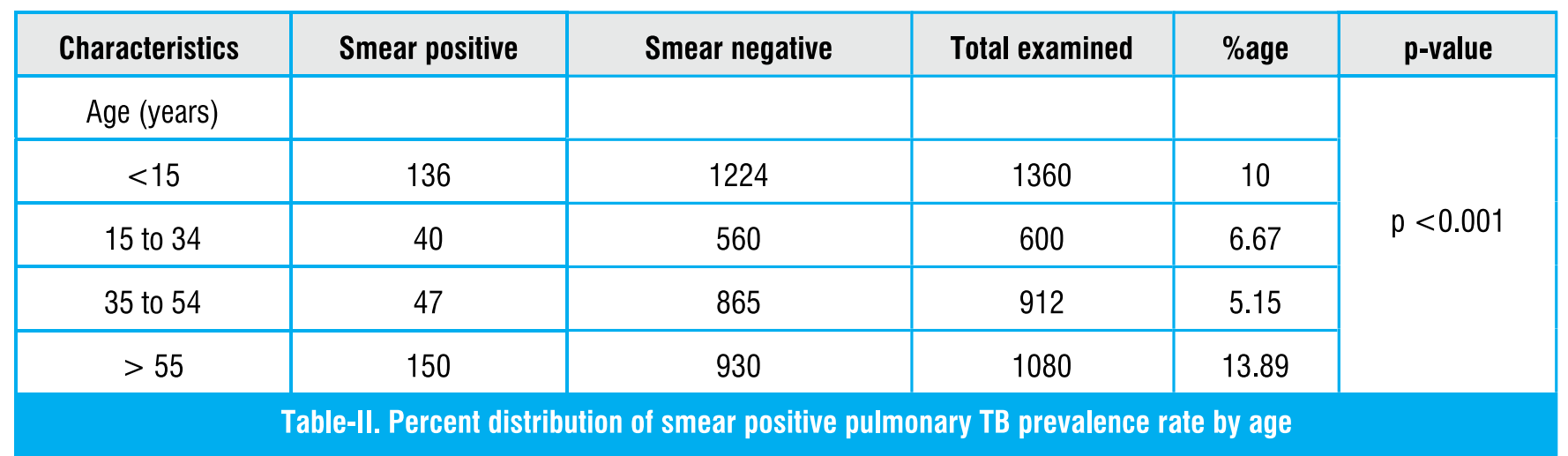

\begin{tabular}{|c|c|c|c|c|c|c|}
\hline & & & \multicolumn{2}{|c|}{ Male } & \multicolumn{2}{c|}{ Female } \\
\hline & Total examined & Positive (\%) & Examined & Positive (\%) & Examined & Positive (\%) \\
\hline Year & & & & & & \\
\hline 2007 & 550 & $52(9.45 \%)$ & 290 & $30(10.34 \%)$ & 260 & $22(8.46 \%)$ \\
\hline 2008 & 735 & $68(9.25 \%)$ & 401 & $40(9.98 \%)$ & 334 & $28(8.38 \%)$ \\
\hline 2009 & 810 & $73(9.01 \%)$ & 485 & $37(7.628 \%)$ & 325 & $36(11.07 \%)$ \\
\hline 2010 & 702 & $69(9.82 \%)$ & 399 & $40(10.02 \%)$ & 303 & $29(9.57 \%)$ \\
\hline 2011 & 605 & $57(9.42 \%)$ & 402 & $30(7.46 \%)$ & 203 & $27(13.30 \%)$ \\
\hline 2012 & 550 & $54(9.81 \%)$ & 350 & $32(9.14 \%)$ & 200 & $22(11 \%)$ \\
\hline Total & 3952 & $373(9.44 \%)$ & 2327 & $209(8.98 \%)$ & 1625 & $164(10.09 \%)$ \\
\hline
\end{tabular}

Table-III. Year specific distribution of number of sputum smear examined and positive results from January 2007 to December 2012

a systematic manner on quarterly bases. National TB control programme ${ }^{3}$ integrates and focuses in providing diagnostic and treatment facilities in public sector. Current global TB control policy emphasizes passive case finding through sputum smear microscopy for patients who self-report to primary health centers. Although WHO has recommended certain laboratories in private sectors as DOTS diagnostic centers, however data collection from these centers and other private sector laboratories is very erratic. Patient diagnosed in these centers may not be registered under DOTS programme and are therefore not included while calculating the prevalence of pulmonary tuberculosis.
In our study; overall smear positive pulmonary tuberculosis is found to be above $9 \%$. The prevalence rate is similar to a study conducted in a teaching hospital Ethiopia ${ }^{5}$. The cases reported in our study are not included in the data collected in national TB control programme.

One of the components of stop TB strategy is to engage all health care providers ${ }^{4,7,8,12}$ including publicpublic and public-private mix (PPM) approach. However our study shows that the reports submitted to WHO refers only to patients treated under DOTS; there is little information about the fate of patients treated outside these programmes. The reporting of 
new cases may not be complete because there is no mandatory reporting from the private sector that form a sizeable proportion of health care provider system. Most of the laboratories have very poor record keeping of the smear positive cases. None of these laboratories have ever admitted any report of new case detection to health authorizes.

\section{CONCLUSIONS}

It is concluded that efforts to collect data from WHO recommended DOTS diagnostic centers outside the public sectors is not regular. Therefore a large number of sputum smear positive cases remain unregistered with DOTS and also not included in calculating the prevalence of the disease. We recommend that a comprehensive review of the national TB control programmed should be taken. It requires reorientation to include the mandatory case notification by all health care providers.

\section{Copyright(C) 20 Aug, 2013.}

\section{REFERENCES}

1. A De Muynck, S Siddiqi, A Ghaffar, H Sadiq. Tuberculosis control in Pakistan: critical analysis of its implementation. JPMA January, 2001.

2. Wise J. WHO identifies $\mathbf{1 6}$ countries struggling to control tuberculosis. BMJ 1998 Apr 11:316(7138): 1113 [Pub Med].

3. Zumla A, Mwaba P, Huggett J, et al. Reflection on white plague. Lancet Infect Dis 2009;9(3) 197-202 [pub Med].
4. National TB control programme. www.ntp.gov.pk/ about.htm.

5. Global tuberculosis report 2012. WHO/HTM/2012.6.

6. Ali H, Zeynudin A, Mekonnen A, Abera S, Ali Si. Smear positive pulmonary tunerculosis(PTB) prevalence amongst patients at Agaro teaching health center, South West Ethiopia. Ethiop J Health Sci.2012 Mar;22 (1):71-6. [pub Med].

7. TB prevalence survey: a handbook, Geneva, World Health Organization. 2011 (WHO/HTM/TB/2010.17).

8. The global plan to stop TB, 2011-2015, Geneva, World Health Organization, 2010(WHO/HTM/STB/2010.2).

9. Dye C, Maher D, Weil D, Espinal M, Raviglione M. Targets for global tuberculosis control. Int J Tuberc Lung Dis 2006 Apr 10(4):460-2, stop TB department, World health Organization, Geneva, Switzerland. dyec@who.int.

10. Pio A, Leulmo F, kumaresan J, Spinaci S, National tuberculosis programme review: experience over the period 1990-95. Source National Programme support, global tuberculosis programme, World Health Organization, Geneva, Switzerland.

11. WHO policies on TB diagnostics are available at: www. who.int/tb/laboratory/policy_statement

12. Javid A. Tuberculosis control in Pakistan (editorial) PJCM.net/pdf_v16_n4_e1.pdf.

13. Khan M. Ibrahim, Samreen Khan, Ulrich Laaser. Tuberculosis: Current status, challenges and barriers ahead in 22 high endemic countries. www.ayub med.edu.pk/JAMC/PAST/14-4/ibrahim.htm.

\section{AUTHOR(S):}

1. DR. SAEED AKRAM BHATTI MBBS, FCPS (Medicine) Assistant professor Pharmacology, Sargodha Medical College

2. DR. ABDUL HASEEB KHAN MBBS, DCP, M Phil (Histo) Assistant professor Pathology, Sargodha Medical College

3. DR. MAQBOOL ALAM MBBS, MCPS, FCPS Consultant Haematologist
Correspondence Address:

Dr. Saeed Akram Bhatti

dr.saeed@uos.edu.pk drsaeedakram@yahoo.com.pk 